\title{
Are Mental Disorders Brain Diseases and What Does This Mean? N'Famory Camara ${ }^{* *}$ and Emmanuel Binyet ${ }^{2}$
}

${ }^{1}$ Department of Economics/Management Science and Engineering, University of Geosciences, Beijing, China

${ }^{2}$ Department of Power Mechanical Engineering, National Tsing Hua University, Taiwan

\begin{abstract}
The aim of this paper is to increase awareness of the prevalence of neurological disorders. To do so, we highlight via in-depth literature review the distinction between mental disorders, brain diseases and psychiatric disorders. This distinction will give a holistic view into the disorders. This in turn will facilitate the suggestion of reasonable conclusions that can enhance the way in which mental disorders or brain diseases are being diagnosed and treated.
\end{abstract}

Keywords: Brain diseases; Mental disorders; Recalcitrant environment; Etiology

\section{Introduction}

Mental diseases, so claims go, should be understood and treated as brain disorders [1]. Disease and disorder should be understood in terms of morbid anatomy and physiology. Eventually, this will help to generate causal hypotheses about psychiatric disorders in terms of neuropathology or dysregulation. Nonetheless, in the context of neurobiological pathology, psychiatric disorders are identifiable as disorders of the brain [2]. According to neuropsychology and experimental neuroscience, mental functions are specifically located in the brain, thus mental dysfunctions may be traced back to abnormal brain areas as well $[3,4]$. There is evidence that aspects of mental functions can be reliably associated with specific brain areas, as revealed by neuroimaging experiments in healthy subjects or by lesiondeficit correlations in neurological patients [5]. This shift supported by evidence from neuroimaging studies, allows to look beyond the lesion and to identify viable brain networks with capacity for plasticity [6]. However, mental dysfunction may involve lesions or functional abnormalities of the brain, but also, some maladaptive operating rules acquired by learning. This is to suggest that the brain generates mental abilities and mental disorders [7], due to defective computational principles [8]. Thus all mental illnesses are cerebral illnesses [9]. This statement, according to Jaspers, is nothing but a set of beliefs [10]. In some loose sense, it is very likely that everything is dogma. Since believing in a science suggests that the latter science is in fact based on a set of beliefs (e.g., reality, logic or norm). These beliefs can only be altered by new studies or finding that could claim better ways or beliefs. Similarly, Dr. Insel states that mental disorders are brain disorders [11]. He suggests that problems like mood disorders and anxiety are caused by identifiable brain abnormalities. Furthermore, Insel commented on this position by revealing that mental disorders are not caused by brain lesions as with neurological disorders [12]. But they are rather from abnormal development of brain circuitry. One further reason that mental disorders are portrayed as brain diseases by researchers, despite failing to fulfill the primary definition of a disease: is that a deviation in bodily architecture or behavior is the reductionist philosophy that unusual psychological phenomena are the result of a disordered brain. Marco Stier argues in this way that mental disorders can only be determined on the mental level since behavior can only be termed deviant by comparing it to the norms of non-deviant behavior [13] However, from the above basics, in the following section we will try to illustrate different scenarios that could give us significant insights into the disorders' etiology. This in turn will help to provide enhancement into how disorders related to brain are being diagnosed and treated.

\section{Discussion}

It is likely that for a behavior to be deviant or non-deviant, it largely depends on what the eyes are seeing and transmitting to the brain. For instance, suppose that two individuals are put in different cages; the first cage contains a wild animal and the second cage contains a trained animal. It is obvious that the one facing the wild animal would have a more deviant behavior than the second one. This demonstrates that the environment is not just an input; it is an essential part of the cognitive processing. Thus, a brain-side story alone is insufficient to explain the mechanism underpinning solution of the cognitive task. Here is an example of the intended explanatory contrast. Consider the task of catching a fly ball in baseball [14]. The mechanism involved in successfully catching a baseball is not wholly neural; the mechanism rather involves neural activity working together with the environment in a loop. Another example is how players select their words in Scrabble [15].

A brain scanner, even if connected to a player during a play, would not be able to reveal the mechanism behind the player's word choice. Therefore, a behavior, thought, or belief can be the result of an on-going loop between brain, body, and environment. In these cases, an explanation of how a behavior, thought, or belief tends to occur involves the whole mechanism. To find consistence with this, it would be interesting to investigate how mental disorders are characterized with blind and deaf people; as these people can't see or hear. Therefore evaluating how the interaction between brain and environment is manifested among these people (blind and/or deaf) could give new interesting insights. As Camara and Binyet argue that the amount of mental disorder in developed countries seems higher than in developing countries due to ICT factors [16]. In this manner, it is very probable that proportionally the amount of mental disorders among people with functioning eyes and ears or only having functioning eyes but being deaf is higher than the amount of mental disorders among blind people due to environmental and ICT factors. In this sense, it is also very likely that the amount of mental disorders among people with functioning eyes

*Corresponding author: N'Famory Camara, Department of Economics/ Management Science and Engineering, China University of Geosciences, Beijing, China, Tel: 008415072444291; E-mail: nfamoryc@yahoo.fr

Received August 10, 2017; Accepted August 16, 2017; Published August 21 2017

Citation: Camara NF, Binyet E (2017) Are Mental Disorders Brain Diseases and What Does This Mean? (TDR). Brain Disord Ther 6: 239. doi: 10.4172/2168975X.1000239

Copyright: (c) 2017 Camara NF, et al. This is an open-access article distributed under the terms of the Creative Commons Attribution License, which permits unrestricted use, distribution, and reproduction in any medium, provided the original author and source are credited. 
and ears is lower than those with functioning eyes but being deaf. This is because deafness is a heterogeneous condition with far-reaching effects on social, emotional, and cognitive development [17]. Furthermore, investigators using a sign-language-based interview in Sweden noted that deaf older people had higher rates of depression and insomnia than did hearing individuals. Fellinger and colleagues reviewed the extensive evidence of mental health problems in deaf people, which are substantially more common than in hearing populations [18].

Increased rates of mental health problems are reported among deaf people. The language and communication environment of the family is a crucial variable affecting psychosocial wellbeing of deaf children. This shows somehow that sight and the hearing are crucial components in mental illness. Let us get back to that deviant and non-deviant behavior. Suppose again that two individuals are put in different cages (one blind and one deaf). Suppose this time that both cages contain a wild animal each. It is obvious that the deaf one will have a more deviant behavior than the blind one. Why is this so? It is simply because of what the eyes of the deaf individual transmit to his brain. The hearing could also cause a deviant behavior (e.g., hearing a terrifying noise). But from these above examples, it is more likely that sight could be a greater transmitter of mental disorders than hearing. In other words, the amount of deaf people with mental disorders must be higher than the amount of blind people affected with mental disorders. This makes sense because deaf people are facing several factors that contribute to high prevalence of mental disorders. For instance, Fellinger and coworkers report about the social hardship coupled with deafness, the high prevalence of anxiety and depression among deaf people, and the obstacles they face in profiting from mental health services [19].

To that, we can add that the sight is a big transmitter of thoughts. Although, seeing is better than being blind but there are serious implications when one confronts a recalcitrant environment. There is evidence that humans rely greatly on vision [20] therefore, serious investigations should be undertaken to evaluate the real contribution of these crucial components (e.g., sight and hearing). Another interesting scholar argues that this view is wrong, and that mental disorders should be identified at the person level, not the brain [2]. This is to suggest that the things that are going wrong in mental disorder are picked out at the person-level. In the light of this, suppose a new born with a stomach ache. The question here is how the mother would identify the illness since the baby does not yet have the ability to speak. Well, sure thing is that the mother can only notice this illness at the new born level (e.g., repeated cry or dysentery). Furthermore, suppose a man having any kind of disease such as (chickenpox, malaria or stroke). Superficially, the primary identification would always be at the person level. Internally, the secondary identification would help to localize and classify the disease (whether it is a part of the body or an organ that is affected). Consequently, the assumption that a mental disorder is identified at the person level, not the brain is only a primary identification. Thus, the primary locus of disorder is within a person's own psychological life and interpersonal relationships [21].

Therefore, the brain can be considered as the secondary locus of disorder. For instance, supposing again someone having a stomach ache that goes to the hospital telling the physician that he is suffering from a mental illness. Superficially, the physician could believe his story. But internally, stomach ache and brain disease are not treated at the same level. This situation already presents a disorder. Suppose this time that the same scenario is happening in a non-hospital area with a physician experienced in both stomach and brain diseases. How could the physician still identify what is really wrong with the patient? It is only after superficial interrogation and internal examination diagnosis that the physician would be able to determine the disease. This is to suggest that the medical staff judgment potential also plays a crucial role in identifying the disease. Since there is evidence that some patients had been assigned a diagnosis of depression but actually had suffered from unrecognized infections such as borreliose and hepatitis [22]. If the identification was only at the person-level so how can we explain these mistakes? Does the mistake come from the patient misleading information or is it made at the medical staff level? Or should this be considered a simple misdiagnosis? However, Jaspers argues that to comprehend psychopathology, we should gather insights into the patient's experience, not only for the reasons of treatment but also for holding track of precisely what the phenomena are that we are interested in Mental disorders as "brain diseases" and jaspers' legacy [10].

Like most mental disorders, the diagnostic criteria must involve patient history and behavioral assessment without the availability of laboratory or radiologic confirmation. This is to suggest that the patient and the physician both play a crucial role. The patient's factors include himself, his brain and the environment. While to make a proper diagnosis, the physician must gather information from several sources to evaluate the patient's symptoms in different settings, construct an appropriate differential diagnosis for the presenting complaints, and be familiar with normal development and behavior. In addition, the physician would need skills and knowledge to process those components. To acquire those abilities, it is crucial to encourage efforts by residency programs, medical societies, medical schools, and encouraging medical tutorial programs to augment doctor insights about mental illness and its treatment. Also encouraging doctors and medical groups to work with schools to improve teachers' abilities to recognize mental illness and appropriately recommend parents to seek medical evaluation of potentially affected children. Further study has shown that heritability also plays a role in a disease disorder. For instance, heritability statistics demonstrate that genetic factors account for $75 \%$ to $80 \%$ of the variability perceived in susceptibility to attention deficit hyperactivity disorder and autism, schizophrenia, and bipolar disorder [22]. The statistics also show that genetic factors account for $35 \%$ to $50 \%$ in the case of major depression and alcohol addiction. For other complex disorders, such as breast cancer, Crohn's disease, and diabetes, heritability ranges between $55 \%$ and $70 \%$ [23]. This evidence underlines the finding of formal genetic and candidate gene studies that genetic as well as environmental factors contribute to these disorders. Thus, mental illness is somehow part of our society as Camara and Binyet put forward [16]. This fact is supported by some hidden factors such as heritability or genetics factors, the process of an ageing population, the development of information communication and technology (ICT), drug abuse, and others (e.g., developmental disorders, corruption and malicious activities). Consequently, to eradicate mental disorders or brain diseases, there is a need to control the above hidden factors. A question for further research is if we can do so. Nonetheless, if interpreted uncritically, these studies lead to an inadequate localizationist view of the brain [24], as they do not account for the fact that the brain can reorganize and compensate damage, at least to some extent. For instance, numerous studies e.g., on neurodegenerative disorders have revealed a variety of intraorganismic or intrapersonal factors, such as brain volume, education, socioeconomic status, education etc. [25] which influence the course of the disease [26]. 
Moreover, the notion of cognitive $^{1}$ reserve or brain ${ }^{2}$ reserve can explain epidemiological data revealing that individuals in higher levels of mental and physical activity via occupation, recreation, and education were at lower risk of developing Alzheimer's disease and other forms of dementia [27]. The variety of experimental findings described above suggests that strategies to enhance mental and physical activity levels in the general community should be a priority. Mainstream health promotion can be done via speech and writing through radio, television, leaflets, and websites $[28,29]$. This could help to stimulate people's resilience. Resilience reflects the ability to maintain a stable equilibrium. In the growing literature, resilience is identified in terms of conservative factors that encourage the development of healthy personality characteristics and positive outcomes among children exposed to aversive or unfavorable life circumstances [30,31]. Most people are exposed to at least one violent or life-threatening (e.g., loss or potentially traumatic events) situation during the course of their lives [32]. In this context what about exposed individuals who exhibit relatively little distress? Trauma theorists are sometimes surprised when exposed individuals do not show more than a few PTSD symptoms. For example, in the aftermath of the Oklahoma City bombing, body handlers have been portrayed as displaying unexpected resilience [33]. Further example is the ample majority (78.2\%) of individuals exposed to the 1992 Los Angeles riots who expressed only three or fewer PTSD symptoms [34]. However, resilient individuals could experience transient anxieties in routine functioning (e.g., restless sleep or several weeks of sporadic preoccupation) but generally display a secure trajectory of healthy functioning along time, as well as strong positive emotions and generative experiences [35]. If resilience represents distinct trajectories that are informed by different coping habits, then what factors promote resilience? Meta-analytic reviews have regularly exposed many clear predictors of PTSD reactions, including low intelligence and lack of education, lack of social support, prior psychiatric history, family background, and aspects of the trauma response itself, such as dissociative reactions [36]. An increasing body of testimonial shows that the personality trait of hardiness [37] helps to lessen exposure to extreme stress. Hardiness includes three dimensions: the belief that one can learn and grow from both positive and negative life experiences, the belief that one can influence one's surroundings and the outcome of events, and being committed to finding meaningful purpose in life. Equipped with this set of convictions, hardy persons have been found to minimize potentially stressful situations as less threatening, thus appraising the experience of distress. These beliefs demonstrate in somehow that resilience must be rare and found only in exceptionally healthy people [38]. Another dimension linked to resilience is self-enhancement. Recently, however, research has shown that positive emotions can help reduce levels of distress following aversive events both by quieting or undoing negative emotion [39] and by increasing continued contact with and support from important people in the individual social environment [40]. By contrast, Goldstein followed the ideas of his cousin Ernst Cassirer from 1874-1945, who emphasized that humans have the inherent ability to use symbols in a very productive way [41], in contrast to animals. Thus, humans do not just inhabit environments, but actively create them in terms of language and culture. In the light of this, suppose a patient facing a recalcitrant environment, where he is confronting as stated above some symbols or signs that are really unusual to him and most people in the society. As

${ }^{1}$ Brain reserve corresponds to neuroprotective brain ability, which can be influenced by chronic improvement of mental (and physical) activity [54,55].

${ }^{2}$ Cognitive reserve encloses enhanced complex mental activity, and increased cognitive function, as defensive factors against dementia and other brain disorders [56-58]. stated above some symbols are very productive. Now suppose that this patient is facing some unproductive (frightening) symbols or signs. The input-output picture understands cognitive activity as roughly being a linear flow in which the senses deliver input, which is progressively processed by perceptual and cognitive resources, resulting in an output (usually a motor action), and then the whole process repeats [42]. This means that the more input pictures that the patient will receive from the recalcitrant environment will produce several output pictures (thoughts). Thus an increase in input pictures will correspond to an increase in output pictures. The output pictures (thoughts) would also depend on the quality and quantity of the input pictures (sight) whether, it is a productive or unproductive symbol or sight. Ultimately, a high speed of input-output picture could lead to brain hyperactivity. Attention-Deficit/hyperactivity disorder (ADHD) is a common neuropsychiatric syndrome with onset in childhood, most commonly becoming apparent [43]. In the light of this, the latter patient has a great chance to be victim of mental disorders without relatives and friends noticing a thing. The degree of the patient's resilience and the time spent in that recalcitrant environment are also important factors in the disease growth. All manner of neural malfunctions, when coupled with a recalcitrant environment, may produce similar symptoms [42]. For all these above reasons, diagnosing and treating mental disease should be undertaken very carefully. This is because mental disease or brain disorder can have different etiology. Consequently, it would be helpful to create a national guideline that shows in details these different etiologies and actions to take in order to prevent them. In addition, since the environment plays a crucial role in the disorder therefore it would be interesting to also create a type of pharmaceutical glasses for the patient. These pharmaceutical glasses should have the ability to record whatever the patient is seeing and hearing for a given period. In addition, the prescription of these types of glasses should be anonymous. In other words, the patient should not be aware of the real purpose of the prescription. This would help to avoid biased evaluation. Hence, data collected from the record could give significant insights into how to diagnose and treat disorders mostly related to the environment.

\section{Conclusion}

To summarize, how mental disorders can be classified, identified, diagnosed and treated? To understand this, let us consider how physical, mental and psychiatric disorders are identified. Regarding physical conditions, a condition is classified as disorder when there is assumed dysfunction howsoever explained, whether bio statistically [44] or evolutionarily [45] as alteration from a norm of functioning in the body. While, in the mental context, a condition is classified as disordered when there is a disruption of some kind in the person's feelings, thoughts, or behaviors [2]. Similarly, psychiatric disorders may not have a single identifiable internal malfunction. Rather, they are the product of small, perhaps otherwise untroubling, internal misfunctions, that jostling together, and being reinforced by an uncooperative environment, snowball, and contribute to wider breakdown [42].

These controversies sound with the current recognition that any given mental disorder can be marked by disturbances among several mechanisms, and one singular mechanism may prompt to the psychopathology of a large number of disorders [46]. Nonetheless, the overall approach to diagnosis may involve (a) an exhaustive interview with the patient (e.g., obtaining appropriate testing, performing a careful examination, and necessary multiple histories); (b) a mental state checkup of the patient; (c) a medical assessment for neurologic status and general health; (d) a cognitive evaluation of achievement and ability; (e) 
family and friends reports and other adjunctive evaluations if necessary [47]. This treatment generally will include pharmacotherapy (usually with stimulant medication) along with adjunctive psychoeducation, behavioral therapy, environmental changes, and, at times, supportive psychotherapy of the patient, the family, or both [43]. Nonmedication interventions encompass parent guidance; parent executive training (contingency management in individual or group setting; this method increases parents' self-confidence, lowers troublesome behavior, and diminishes family stress); classroom environmental manipulations; support groups such as children and adults with attention deficit disorder and attention deficit disorder association for families; uncertainty management and daily report cards by teacher; individual psychotherapy for depression, anxiety, and low self-esteem; impulse and social skills control training; and summer treatment programs. Moreover, as stated earlier, the creation and dissemination of practice guidelines for mental diseases designed by appropriate specialists and their use by practicing physicians will assist people to strengthen their resilience. Regarding resilience, a resilient research suggests that mental illnesses lie along a spectrum $[48]^{3}$.

The stark fact is that no one has yet agreed on how best to define and diagnose mental illnesses. Diagnostic and Statistical Manual (DSM-5) of mental disorders, as the two previous editions, put disorders in distinct categories [49] such as Obsessive-Compulsive Disorder (OCD), bipolar disorder, schizophrenia and major-depressive disorder. Since the early 1980s, these categories have guided psychiatry, and are built largely on decades-old theory and subjective symptoms. The problem is that biologists have been unable to find any genetic or neuroscientific evidence to support the breakdown of complex mental disorders into separate categories. Regier and others wanted the latest DSM to move away from the category approach and towards one called 'dimensionality', in which mental illnesses overlap [50]. From this view, the disorders are the result of shared risk factors that escort to abnormalities in intersecting drives such as reward anticipation and motivation, which can be ascertained (hence 'dimension') and utilized to put people on one of several spectra. In other words, Hyman suggests that there is a need for researchers to think outside these traditional silos [51]. They need to re-analyze these conditions from the bottom up. Nonetheless, in the past recent years, some scholars have taken up the challenge and the findings from brain-imaging and genetics studies support the idea that the DSM disorders overlap. This shift via neuroimaging has improved the way in which we view brain diseases. For instance, the ability to determine salvageable tissue, testimonial of changes in remote networks, changes of structural and functional connectivity, and adjustments in cortical thickness are examined in the context of their impact on brain disease [6]. To be precise, neuroimaging techniques can assist to identify connectivity of brain regions and networks. A significant challenge is to identify the brain networks and processes mediating functional improvement so that rehabilitation strategies can be aimed at the appropriate targets. Functional neuroimaging techniques, such as functional MRI (FMRI) and functional task related PET, allow interrogation of changes in brain activity at a whole brain level [6]. They allow monitoring of ongoing brain activity in vivo. They can also be used to localize zones in the brain that are triggered in response to experimentally controlled stimulation or other task achievement. Functional magnetic resonance imaging

${ }^{3}$ In some instances, a spectrum approach puts together states of health that were previously considered separately. A remarkable example of this trend is the autism spectrum, where states of health on this spectrum may now all be referred to as autism spectrum disorders. In other instances, what was treated as a single disorder comes to be seen as comprising a range of types, a remarkable example being the bipolar spectrum.
(FMRI) evaluates brain duty or activity by identifying related changes in blood flow, on the basis that cerebral blood flow and neuronal activation are coupled [52].

The evolution of neuroimaging to permit the examination of network functions is an exciting development to better understand the dynamic process of recovery after disease. Further studies should focus in understanding the real contribution of these components (e.g., sight and hearing) in mental diseases. Although research has established that mental disorders are complex and that their development involves interactions between genetic and environmental factors, their etiology remains largely unknown [53].

\section{References}

1. Insel TR, Quirion R (2005) Psychiatry as a clinical neuroscience discipline. JAMA 294: 2221-2224

2. Banner NF (2013) Mental disorders are not brain disorders. J Eval Clin Pract 19: $509-513$.

3. Kandel ER (1998) A new intellectual framework for psychiatry. Am J Psychiatry 155: $457-469$

4. Kandel ER, Markram H, Matthews PM, Yuste R, Koch C (2013) Neuroscience thinks big (and collaboratively). Nat Rev Neurosci 14: 659-664.

5. Hobson BJA, Leonard JA (2001) Out of its mind: Psychiatry in crisis: A call for reform. Perseus Publishing, USA.

6. Carey LM, Seitz RJ, Parsons M, Levi C, Farquharson S, et al. (2013) Beyond the lesion: Neuroimaging foundations for post-stroke recovery. Future Neurol 8: 507-527.

7. Durling D (1935) Range and distribution of mental abilities in a group of highgrade mental defectives. J Appl Psychol 19: 474-477.

8. Patterson $\mathrm{PH}$ (2002) Maternal infection: Window on neuroimmune interactions in fetal brain development and mental illness. Curr Opin Neurobiol 12: 115-118.

9. Thomas MA, Yue K, Binesh N, Davanzo P, Kumar A, et al. (2007) Localized two-dimensional shift correlated MR spectroscopy of human brain. Magn Reson Med 46: 58-67.

10. Maj M (2013) Mental disorders as "brain diseases" and jaspers' legacy. World Psychiatry 12: 1-3.

11. Deacon BJ, Lickel JJ (2009) On the brain disease model of mental disorders Behavior Therapist 32: 113-118.

12. Moran M (2008) Insel: 'different kind of science' poised to transform psychiatry. Psychiatric Services.

13. Stier M (2013) Normative preconditions for the assessment of mental disorder Front Psychol 4: 611

14. Clark A (2008) Supersizing the mind: Oxford University Press. Philosophical Studies. Oxford, UK 152: 413-416

15. Kirsh D (1995) The intelligent use of space. Artificial Intelligence 73: 31-68.

16. Camara F, Binyet E (2017) Can we eradicate brain disorders or is it just part of our society? Brain Disord Ther 6: 237

17. Fellinger J, Holzinge D, Pollard R (2012) Mental health of deaf people. Lancet 379: $1037-1044$

18. Fellinger J, Holzinger D (2011) Enhancing resilience to mental health disorders in deaf school children. pp. 169-205.

19. Gerich J, Fellinger J (2012) Effects of social networks on the quality of life in an elder and middle-aged deaf community sample. J Deaf Stud Deaf Educ 17 102-115.

20. Barr N, Pennycook G, Stolz JA, Fugelsang JA (2015) The brain in your pocket Evidence that smartphones are used to supplant thinking. Comput Human Behav 48: 473-480.

21. Broome MR, Bortolotti L (2009) Mental illness as mental: In defence of psychological realism. Archives Des Maladies Du Coeur Et des Vaisseaux 52: 1275.

22. Marcella R (2014) Mental disorders are somatic disorders, a comment on $\mathrm{m}$ stier and t. schramme. Front Psychol 5: 53. 
Citation: Camara NF, Binyet E (2017) Are Mental Disorders Brain Diseases and What Does This Mean? (TDR). Brain Disord Ther 6: 239. doi: 10.4172/2168-975X.1000239

Page 5 of 5

23. Sullivan PF, Daly MJ, O'Donovan M (2012) Genetic architectures of psychiatric disorders: The emerging picture and its implications. Nat Rev Genet 13: 537551.

24. Pensinger WL, Paine DA (1980) Development of a mathematical mode of the autogenic brain discharge as spontaneous localization in quantum measurement. Oocities Org.

25. Martin JB (2002) The integration of neurology, psychiatry, and neuroscience in the 21st century. Am J Psychiatry 159: 695.

26. Stern $Y$ (2012) Cognitive reserve in ageing and Alzheimer's disease. Lance Neurol 11: 1006-1012.

27. Nithianantharajah J, Hannan AJ (2009) The neurobiology of brain and cognitive reserve: Mental and physical activity as modulators of brain disorders. Prog Neurobiol 89: 369-382.

28. Gann R (1986) Information services and health promotion. What libraries can do. Health Education Journal 45: 112

29. Quattrin R, Filiputti E, Brusaferro S (2015) Health promotion campaigns and mass media: Looking for evidence. Primary Health Care Open Access 5: 190.

30. Garmezy N (1991) Resilience and vulnerability to adverse developmental outcomes associated with poverty. Am Behav Sci 34: 416-430.

31. Luthar SS, Cicchetti D, Becker B (2000) The construct of resilience: A critical evaluation and guidelines for future work. Child Dev 71: 543-562.

32. Ozer EJ, Best SR, Lipsey TL, Weiss DS (2003) Predictors of posttraumatic stress disorder and symptoms in adults: A meta-analysis. Psychol Bull 129 $52-73$

33. Tucker P, Pfefferbaum B, Doughty DB, Jones DE, Jordan FB, et al. (2002) Body handlers after terrorism in Oklahoma City: Predictors of posttraumatic stress and other symptoms. Am J Orthopsychiatry 72: 469-475.

34. Hanson RF, Kilpatrick DG, Freedy JR, Saunders BE (1995) Los Angeles county after the 1992 civil disturbance: Degree of exposure and impact on mental health. J Consult Clin Psychol 63: 987-996.

35. Bonanno GA, Field NP (2001) Examining the delayed grief hypothesis across five years of bereavement. Am Behav Sci 44: 798-806.

36. Brewin CR, Andrews B, Valentine JD (2000) Meta-analysis of risk factors for posttraumatic stress disorder in trauma-exposed adults. J Consult Clin Psycho 68: $748-766$

37. Kobasa SC, Maddi SR, Kahn S (1982) Hardiness and health: A prospective study. J Pers Soc Psychol 42: 168-177.

38. Casella L, Motta RW (1990) Comparison of characteristics of Vietnam veterans with and without posttraumatic stress disorder. Psychol Rep 67: 595-605.

39. Fredrickson BL, Levenson RW (1998) Positive emotions speed recovery from the cardiovascular sequelae of negative emotions. Cognition and Emotion 12 $191-220$

40. Bonanno GA, Keltner D (1997) Facial expressions of emotion and the course of conjugal bereavement. J Abnorm Psycho 106: 126-137.
41. Goldstein K (1939) The Organism: A holistic approach to biology derived from pathological data in man. New York, American Book Company, USA.

42. Sprevak M (2011) Neural sufficiency, reductionism, and cognitive neuropsychiatry. Philo Psychiatry Psychol 18: 309-328.

43. Wolraich M, Brown L, Brown RT, Dupaul G, Earls M, et al. (2011) Adhd: Clinica practice guideline for the diagnosis, evaluation, and treatment of attentiondeficit/hyperactivity disorder in children and adolescents. Pediatrics 128: 1007 1022

44. Boorse C (1997) A rebuttal on health. In what is disease? (1-134) Humana Press, Totowa, USA

45. Wakefield JC (1992) Disorder as harmful dysfunction: a conceptual critique of DSM-III-R's definition of mental disorder. Psychol Rev 99: 232-247.

46. Sanislow CA, Pine DS, Quinn KJ, Kozak MJ, Garvey MA, et al (2010) Developing constructs for psychopathology research: Research domain criteria. J Abnorm Psychol 119: 631.

47. Barkley RA (1990) Attention deficit hyperactivity disorder: A Handbook fo diagnosis and treatment. Guilford Press, New York, USA

48. Adam D (2013) Mental health: On the spectrum. Nature 496: 416.

49. Heerlein AL (2014) An approach to dsm-5: A breakthrough in psychiatry? Vertex 25: 18-26.

50. Regier DA, Kuhl EA, Kupfer DJ (2013) The dsm-5: Classification and criteria changes. World Psychiatry 12: 92-98.

51. Hyman SE (2014) Time for new schizophrenia rx. Science 343: 1177.

52. Hubbard IJ, Carey LM, Budd TW, Levi C, McElduff P, et al. (2015) A randomized controlled trial of the effect of early upper-limb training on stroke recovery and brain activation. Neurorehabil Neural Repair 29: 703-713.

53. Loo KMJV, Martens GJM (2007) Genetic and environmental factors in complex neurodevelopmental disorders. Curr Genomics 8: 429-444.

54. Schmand B, Smit JH, Geerlings MI, Lindeboom J (1997) The effects of intelligence and education on the development of dementia: A test of the brain reserve hypothesis. Psychol Med 27: 1337-1344.

55. Kochan NA, Breakspear M, Slavin MJ, Valenzuela M, Mccraw S, et al. (2010) Functional alterations in brain activation and deactivation in mild cognitive impairment in response to a graded working memory challenge. Dement Geriatr Cogn Disord 30: 553-568.

56. Adams $N$ (2010) The effects of cognitive reserve on the rate of cognitive decline in persons with mild cognitive impairment, University of Connecticut Health Center, USA.

57. Andel R, Silverstein M, Kareholt I (2015) The role of midlife occupationa complexity and leisure activity in late-life cognition. J Gerontol B Psychol Sci Soc Sci 70: 314-321.

58. Camara D, Emmunuel B (2017) Understanding household energy use, decision making and behavior in Guinea-Conakry by applying behavioural economics. Renew Sustainable Energy Rev 79: 1380-1391. 